\title{
OPTICAL SPECTRA OF COMPACT OBJECTS
}

\author{
E. M. BURBIDGE
}

University of California, San Diego and Institute of Theoretical Astronomy, Cambridge, England

\begin{abstract}
On the basis of their optical spectra, compact objects are divided into 3 classes according to the probable nature of their energy sources, as follows:

(1) Non-thermal: This class includes Seyfert nuclei, most N-type radio galaxies, and also very luminous Zwicky compact objects. The existence of high-velocity clouds, and a wide variety of densities and temperatures appear to be common characteristics of these objects. QSOs are considered to be related, with similar but more extreme properties.

(2) Hot, massive stars: This class includes some small galaxies, parts of galaxies or appendages to galaxies, which are usually much less luminous than class (1) objects.

(3) Stellar: Some compact galaxies having only absorption line spectra fall into this class.

A variety of morphological forms exists amongst class (1) and (2) objects.

Emission line spectra of QSOs are reviewed, and related to the physical conditions in the emitting regions.
\end{abstract}

\section{Introduction}

The widespread interest that exists today in compact extragalactic objects has developed in the past seven years or so, and stems largely from:

(a) the discovery of the quasi-stellar objects;

(b) the survey work of Zwicky $(1964,1966 ; 1964-68)$ which disclosed that an abundant population of compact objects exists in space, following the survey work of Haro (1956) and Markarian $(1967,1969)$ which selected objects with abnormally strong UV continua and which yielded some of the same kind of compact galaxies;

(c) the continuing work on identification and spectroscopy of radio galaxies, which include compact objects.

The earliest work on compact objects was of course that by Seyfert (1943), in his original isolation of what are now known as the Seyfert galaxies. As we shall see, the term 'compact' is a loose descriptive term, and the compact objects in Zwicky's definition comprise compact parts of galaxies as well as compact galaxies, e.g. the 'Ambartsumian knot' in NGC 3561 (Zwicky and Humason, 1961; Zwicky, 1966; Stockton, 1968). His definition, however, excludes the QSOs, because he requires the objects to be just distinguishable from stars on the 48-in. Palomar Schmidt plates.

In any case, Zwicky's lists contain a heterogeneous collection of objects. We shall take it that the definition of 'compact' has partly an operational significance, signifying a galaxy or part of a galaxy with a surface brightness exceeding some assigned value, and partly a deductive physical significance, following from properties such as short-term variability and implied physical conditions. Thus the family of compact objects comprises QSOs, Seyfert nuclei, N-type galaxies, and compact galaxies or parts of galaxies as categorized by Zwicky.

Both Morgan (1970) and Ambartsumian (1970) have emphasized the importance of a morphological approach to the study of these objects, so that the important and 
physically meaningful correlations between the various types of observations can be found and so that ultimately a satisfactory theory embracing all the data can be formulated. Morgan (this volume, p. 97) has described a classification scheme for these objects, with criteria for classification and selected standards for the form classes, and the present paper is intended to be read with that scheme kept always in mind as a basic morphological classification which minimizes observational selection effects due to distance.

I want to make a simple division of my subject matter along physical lines, and first I divide the compacts in Zwicky's sense into three classes:

(1) The first class consists of objects with an energy source of very small dimensions, having a large and often variable energy output generally believed to be of nonthermal origin. The ultimate energy source is probably gravitational energy, but its nature is not fully understood, and other hypotheses, e.g. that by Ambartsumian (1958, 1965), have been advanced. With a non-thermal source, high-energy particles provide the means of transferring energy from the source to what is seen - in our case, either short-wavelength radiation which ionizes the gas and/or the line emission itself. This class includes the Seyfert nuclei and most $\mathrm{N}$-type radio galaxies, and it has a direct link with the QSOs which have similar but more extreme properties. The Seyfert galaxy II Zw $0430+05 \equiv 3 \mathrm{C} 120$ is an example. The class is distinguished partly by its line spectrum, which has strong, broad emission lines, arising from ions going up to a high ionization potential. We shall, however, see later that some N-type radio galaxies have fairly narrow emission lines, yet the radio emission requires a non-thermal source and places them in this class.

(2) The second class consists of small galaxies, parts of galaxies, or appendages to galaxies, and is distinguished from class (1) by the line spectra, which display sharp emission lines resembling bright high-excitation HII regions like the Orion Nebula. The physical distinction from class (1) is that the energy source appears to be hot, massive stars - gigantic $\mathrm{O}$-associations. There is no evidence for non-thermal emission.

(3) Some compact galaxies have only absorption-line spectra, with no emission lines to indicate the presence of hot gas. Such objects must consist only of stars, and the star density must be high. It is not known how common this class is, and little quantitative work has been done on them.

The division of the Zwicky compacts and those Markarian and Haro galaxies which fall in this category into three classes has been made possible by the slit spectroscopic or objective prism studies by Haro, Markarian, and Zwicky themselves, by Arp et al. (1968), DuPuy (1968), Weedman and Khachikian (1968, 1969), Sargent (1970a, b, c), and Arakelian et al. (1970).

Section 2 will cover the classical Seyfert galaxies, $\mathrm{N}$-type galaxies, and compacts of class (1); compacts of classes (2) and (3) will be covered in less detail in Sections 3 and 4, respectively. Finally, Section 5 will discuss rather briefly the emission-line spectra only of the QSOs; the paper by Lynds (this volume, p. 127) will cover the absorption-line spectra of QSOs. 


\section{Seyfert and N-Type Galaxies; Compacts of Class (1)}

\subsection{SeYfert GalaXies}

A fuller account of these than is possible here can be found in Chapters 5, 8, and 9 of the review article by G. Burbidge (1970). Following the original paper by Seyfert (1943), the first general discussion of the observations which attempted to put them in a physical framework was that by Burbidge et al. (1963), on violent events in the nuclei of galaxies. Here the phenomena were related to other evidence for explosive activity in radio galaxies, etc. Many more recent papers and a useful bibliography were published in the Proceedings of a symposium on Seyfert galaxies (Pacholczyk and Weymann, 1968).

Table I lists the bright galaxies known to fall into Seyfert's category, and fainter more distant compact objects found by the various surveys, and discussed in Section 2.3. Radio galaxies classified as $\mathrm{N}$-type are not included here as they are listed in Table III.

Seyfert galaxies are defined by the following properties:

(a) They have a bright star-like nucleus, unresolved optically. Danielson et al. (1968) found that the main luminosity in NGC 4151 is coming from a region with an upper limit of 0 ". 18 for its half-intensity angular diameter;

(b) The spectra show broad Balmer emission lines, often having a narrower core and very broad wings; the forbidden lines can be broad or narrow;

(c) The emission lines include transitions in ions of higher ionization potential than those found characteristically in Hur regions in galaxies, e.g. [Ne v].

Even this subdivision of the class (1) of compact objects is itself a somewhat heterogeneous grouping; de Vaucouleurs and de Vaucouleurs (1968) distinguished between Seyferts with spiral-arm outer parts and those without, and even Seyfert's original list contains three distinctly different types, exemplified by the best-studied objects, NGC 1068, NGC 1275, and NGC 4151. Table I demonstrates this diversity in another way, in the several different kinds of surveys which have yielded Seyfert galaxies: as well as those already mentioned there are the atlases and catalogues by VorontsovVelyaminov (1959) and Arp (1966) and the radio-source catalogues.

Since NGC 1068, 1275, and 4151, from Seyfert's classical list, have been much studied and demonstrate distinct differences from one another, it is worthwhile describing their properties in more detail. For a more complete bibliography covering these and other objects, see G. Burbidge (1970).

\subsubsection{NGC 1068}

This galaxy has a bright main body and well-developed spiral arms, and its rotation has yielded a mass within $2 \mathrm{kpc}$ of the center of $3 \times 10^{10} M_{\odot}$ (Burbidge et al., 1959). NGC 3227 and NGC 7469 resemble it in having fairly bright spiral arms, and the masses of these are similar $-3.5 \times 10^{10} M_{\odot}$ (Rubin and Ford, 1968) and $1 \times 10^{10} M_{\odot}$ (Burbidge et al., 1963), respectively. The nucleus of NGC 1068 is not as concentrated as in some Seyferts; spectra show absorption lines presumably due to the stellar 
TABLE I

Seyfert galaxies and extragalactic objects with compact nuclei and broad emission lines

\begin{tabular}{|c|c|c|c|}
\hline No. & $z$ & No. & $z$ \\
\hline \multicolumn{2}{|l|}{ NGC } & \multicolumn{2}{|l|}{ Markarian } \\
\hline 1068 & 0.0038 & 9 & 0.038 \\
\hline 1275 & 0.0180 & 10 & 0.029 \\
\hline $1566^{1}$ & 0.0039 & 34 & 0.0507 \\
\hline 3227 & 0.0034 & 42 & 0.024 \\
\hline 3516 & 0.0092 & 50 & 0.023 \\
\hline $3783^{2}$ & 0.0093 & 69 & 0.076 \\
\hline 4051 & 0.0023 & 79 & \\
\hline 4151 & 0.0033 & 105 & \\
\hline 5548 & 0.0166 & 106 & \\
\hline $6814^{3}$ & 0.0053 & 110 & \\
\hline 7469 & 0.0166 & 124 & \\
\hline \multicolumn{2}{|l|}{ Zwicky } & 141 & \\
\hline III $0008+10$ & 0.089 & 142 & \multirow{6}{*}{0.070} \\
\hline $0039.5+4003^{4}$ & 0.1026 & $205^{8}$ & \\
\hline I 0051 + 12 & 0.061 & 231 & \\
\hline II $0119-01$ & 0.054 & 273 & \\
\hline I $1535+55^{5}$ & 0.0386 & 279 & \\
\hline II $2130+09$ & 0.061 & 290 & \\
\hline \multicolumn{2}{|c|}{ Vorontsov-Velyaminov (1959) } & Radio & \\
\hline $144^{6}$ & 0.021 & $3 \mathrm{C} 120^{9}$ & \\
\hline $150^{7}$ & 0.027 & OQ $208^{10}$ & \\
\hline
\end{tabular}

\section{References to Table I}

NGC galaxies mostly in Seyfert (1943) and redshift catalogues. Published references for Zwicky and Markarian galaxies given at end of Section 1; unpublished identifications kindly provided by $E$. Khachikian and M.-H. Demoulin, to be published elsewhere. Additional references:

1. G. de Vaucouleurs: 1961, Mem. Roy. Astron. Soc. 68, 69.

2. T. Page: 1967 , Astron. J. 72, 821.

3. M.-H. Demoulin, private communication.

4. F. Zwicky et al.: 1970, Publ. Astron. Soc. Pacific 82, 93.

5. J. deVeny and R. Lynds: 1969, Publ. Astron. Soc. Pacific 81, 535.

6. G. and M. Burbidge: 1964, Astrophys. J. 140, 1307.

7. G. and M. Burbidge: 1961, Astron. J. 66, 541.

8. Weedman, D. W.: 1970.

9. M. Burbidge: 1967, Astrophys. J. Letters 149, L51; W. Sargent: 1967, Publ. Astron. Soc. Pacific 79, 369 .

10. This paper, Section 2.4, p. 120.

content, as well as the Balmer and forbidden emission lines which are both broad, though not as broad as the $\mathrm{H}$ lines in NGC 4151.

Osterbrock and Parker (1965) and Dibai and Pronik (1965) studied the physical conditions in the gas giving rise to the line emission. These are: 


$$
\begin{aligned}
T_{e} & \simeq 9000 \mathrm{~K} \\
N_{e} & >4 \times 10^{3} \text { from [OII] } \\
& \left.>4 \times 10^{4} \text { from [S } \mathrm{SI}\right] \\
& \approx 2 \times 10^{5} \text { from [ArIV] } .
\end{aligned}
$$

As in all such objects that have been studied in sufficient detail to reveal their physical properties, it has been found that very strong density fluctuations must be present in the emitting gas. Dense, cooler condensations are embedded in a more tenuous hotter gas. Even lines from neutral gas are seen: [OI] $\lambda 6300$ (which is a very characteristic feature in spectra of Seyfert nuclei and related galaxies) and [NI] $\lambda 5199$ (this is similar to the [OII] $\lambda 3727$ transition, ${ }^{4} S^{\circ}-{ }^{2} D^{\circ}$ ).

The Bowen fluorescence lines of permitted OIII in the ultraviolet (excited by HeII $\lambda 304$ and seen in normal planetary nebulae) are definitely absent, and Osterbrock and Parker deduced from this fact that there must be an intimate mixture of neutral and ionized matter, so that He II radiation is absorbed in photoionization before it can reach the main body of gas. This can occur only if there are many sources of ionizing radiation, e.g. shock fronts or thin sheets between cooler regions. The ionization is high but not extreme; [Nev] and [Fe VII] are seen but not $[\mathrm{Fex}]$.

A very interesting feature is the character of the emission lines as seen under moderately high dispersion. Walker $(1966,1968)$ found that immediately outside the very small-diameter continuum source, the broad emission lines break up into a number of distinct separate structures - named by him 'haystack profiles' - which indicate that there are separate cloud complexes moving at several hundred $\mathrm{km} \mathrm{s}^{-1}$ relative to one another.

\subsubsection{NGC 1275}

This Seyfert galaxy, the brightest member of the Perseus cluster, is the well-known strong radio source with a very interesting radio structure, including a component of extremely small angular diameter at the center, and outer structures that involve nearby radio-source galaxies in the Perseus cluster. NGC 1275 is also famous for having two distinct emission-line redshifts, discovered by Minkowski (1957). The main body of the galaxy has a recession velocity of $5300 \mathrm{~km} \mathrm{~s}^{-1}$, and an outer filamentary structure on one side of the object has a velocity of some $8300 \mathrm{~km} \mathrm{~s}^{-1}$. The velocity field was mapped out by Burbidge and Burbidge (1965) and was interpreted as being due to an explosive event $\sim 10^{6} \mathrm{yr}$ ago in the nucleus. This ejected a large body of gas asymmetrically. Narrow-band filter photography by Lynds (1970) showed that, in addition to the gas ejected at about $3000 \mathrm{~km} \mathrm{~s}^{-1}$, the gas at velocities close to the systemic velocity is distributed around the galaxy in a filamentary structure, looking very like a gigantic Crab Nebula.

Dibai and Pronik (1966) studied the physical conditions in the line-emitting gas. The mass of the gas ejected at $3000 \mathrm{~km} \mathrm{~s}^{-1}$ is some $10^{8} M_{\odot}$. Although the main galaxy has often been called an elliptical, Minkowski (1968) showed that there are 
Balmer absorption lines in its spectrum, so that early-type stars must be present and the stellar population must thus be younger than that characteristic of ellipticals.

\subsubsection{NGC 4151}

This object is representative of the commonest type of Seyfert galaxy. Although classified as a spiral, its spiral arms are rather irregular and ill-defined, and are much fainter relative to the nucleus than is the case in NGC 1068. The Balmer emission lines are very broad, with a half-intensity half-width of some $3000 \mathrm{~km} \mathrm{~s}^{-1}$, and they have a narrower core. The forbidden lines have profiles like the hydrogen cores. These cores show the 'haystack' profiles demonstrating the existence of fast-moving cloud complexes of ionized gas (Walker, 1968).

The most detailed spectrophotometric study is by Oke and Sargent (1968). The gas producing the emission lines is hotter than that in NGC 1068, with $T_{e} \simeq 18000 \mathrm{~K}$ and $N_{e} \simeq 5 \times 10^{3} \mathrm{~cm}^{-3}$. In this object also, extreme density and temperature fluctuations must exist, because lines of $\left[\mathrm{OI}_{1}\right]$ and of [Fe VII], [Fex] are present, and Oke and Sargent suggested the identification of [FexIv] $\lambda 5303$ also. This might arise in a hot low-density gas at $T_{e} \simeq 10^{6} \mathrm{~K}, N_{e} \simeq 10^{2} \mathrm{~cm}^{-3}$, which would give pressure equilibrium with the cooler, denser regions. Table II lists the lines identified by Oke and Sargent.

The extreme range of ionization poses a problem. Williams and Weymann (1968) and Osterbrock (1969) discussed the line identifications and strengths, and deductions therefrom. Shock waves produced in collisions of high-velocity clouds will produce high-temperature regions; Shklovsky (1966) suggested that there might be another component of ionizing radiation, in addition to the far UV tail of the known UV component, which might give a rise in the continuum flux in the $\mathrm{X}$-ray region and which could produce the ions of highest ionization potential.

Williams and Weymann pointed out that the Bowen fluorescence lines of [O III], which are not seen in NGC 1068, do appear in NGC 4151, but are much weaker than in planetary nebulae. Regarding the abundances of the elements, the line intensities calculated by these authors were derived on the assumption of 'normal', solar-neighborhood, relative abundances. Osterbrock (1970) noted, however, that helium appears to be underabundant in this nucleus.

\subsubsection{Emission-Line Widths in Spectra of Seyfert Nuclei}

The question of the line-broadening mechanism that produces the great widths of the emission lines in spectra of Seyfert nuclei is still not settled. These could be due to electron scattering or to Doppler broadening by either random or organized motions. Electron scattering was suggested by Burbidge et al. (1966) for the broadening of emission lines in spectra of QSOs, and for NGC 4151 it was suggested by Oke and Sargent (1968). Williams and Weymann (1968), however, found difficulties with this hypothesis.

It is true that the 'haystack' profiles in NGC 1068 and 4151 demonstrate that part, at least, of the broadening is due to large random velocities of the gas. The very extended wings in the permitted lines in some Seyfert nuclei, however, are quite 
TABLE II

Emission lines in nucleus of NGC 4151 : Identifications, intensities, and comparison with the planetary nebula NGC 7027, according to Oke and Sargent (1968)

\begin{tabular}{|c|c|c|c|c|c|}
\hline \multirow[t]{2}{*}{$\lambda(\AA)$} & \multirow[t]{2}{*}{ Identification } & \multirow[t]{2}{*}{$\begin{array}{l}\text { Equivalent } \\
\text { width }(\AA)\end{array}$} & \multirow{2}{*}{$\begin{array}{l}\text { Flux at source } \\
\text { (units of } 10^{40} \\
\mathrm{erg} \mathrm{s}^{-1} \text { ) }\end{array}$} & \multicolumn{2}{|c|}{$\begin{array}{l}\text { Strength relative to } \\
\mathrm{H} \beta=100\end{array}$} \\
\hline & & & & NGC 4151 & NGC 7027 \\
\hline 10830.2 & HeI & 163.5 & 5.98 & 81 & 87 \\
\hline 10049.4 & $\mathrm{P} \zeta$ & 30.0: & 1.19: & 16: & 5 \\
\hline $\left.\begin{array}{l}7329.9 \\
7330.7\end{array}\right\}$ & [OII] & 11.0 & 0.62 & 8 & 32 \\
\hline 6731.3 & {$\left[\mathrm{~S}_{\mathrm{II}}\right]$} & 33.3 & 2.08 & 28) & 6 \\
\hline 6717.0 & [SII] & 27.7 & 1.75 & $24\}$ & \\
\hline 6583.6 & [NII] & 29.0 & 1.83 & 25 & 90 \\
\hline & $\int \mathrm{H} \alpha$ wings & 360.0 & 22.75 & 307) & \\
\hline 6562.8 & $\{\mathrm{H} \alpha$ core & 34.0 & 2.15 & $29\}$ & 290 \\
\hline 6548.1 & {$\left[\mathrm{~N}_{\mathrm{II}}\right]$} & 6.2 & 0.39 & 5 & 30 \\
\hline 6374.5 & {$[\mathrm{Fex}]$} & 2.8: & 0.18 & 2: & - \\
\hline 6363.9 & [OI] & 4.7 & 0.31 & 4 & 6 \\
\hline 6300.2 & [OI] & 20.6 & 1.36 & 18 & 20 \\
\hline 6085.3 & [Fe VII] & 7.6 & 0.52 & 7 & - \\
\hline 5875.6 & HeI & 4.4 & 0.31 & 4 & 11 \\
\hline 5754.8 & {$[\mathrm{NII}]$} & 3.0: & $0.22:$ & 3: & 8 \\
\hline 5720.9 & [FevII] & 4.4 & 0.32 & 4 & - \\
\hline 5303.6 & [FexIV] & 1.0: & 0.08 & 1: & - \\
\hline 5006.8 & [OIII] & 188.0 & 15.80 & 214 & 1460 \\
\hline 4959.9 & [OIII] & 62.0 & 5.23 & 70 & 480 \\
\hline 28613 & $\int \mathrm{H} \beta$ wings & 72.0 & 6.07 & 82) & 100 \\
\hline 4861.3 & $\{\mathrm{H} \beta$ core & 16.0 & 1.35 & $18\}$ & 100 \\
\hline 4799.5 & [Fe III] & 1.0: & 0.10 : & 1: & - \\
\hline 4740.3 & [AIV] & 1.7 & 0.15 & 2 & 10 \\
\hline 4711.4 & [AIV] & 1.7 & 0.15 & 2 & 8 \\
\hline 4685.7 & HeII & 21.7 & 1.88 & 25 & 46 \\
\hline 4658.1 & [FeIII] & 5.9 & 0.51 & 7 & - \\
\hline 4471.5 & HeI & 1.0: & 0.10 : & 1: & 4 \\
\hline 4363.2 & [OIII] & 5.4 & 0.48 & 7 & 26 \\
\hline & $\int \mathrm{H} \gamma$ wings & 21.4 & 1.92 & 26) & \\
\hline 4340.5 & H $\gamma$ core & 5.6 & 0.50 & $7 j$ & 47 \\
\hline 4243.0 & $?$ & 0.5 : & 0.04: & 1: & - \\
\hline 4228.0 & ? & 0.5 : & 0.04: & 1: & - \\
\hline & ( $\mathrm{H} \delta$ wings & 5.4 & 0.51 & 7) & \\
\hline 4101.7 & $\{\mathrm{H} \delta$ core & 3.7 & 0.35 & $5\}$ & 26 \\
\hline 4076.2 & [SII] & 2.3 & 0.22 & 3) & \\
\hline 4068.6 & [SII] & 3.5 & 0.34 & $5\}$ & 16 \\
\hline $\begin{array}{l}3970.1 \\
3968.5\end{array}$ & $\left.\begin{array}{l}\mathrm{H} \varepsilon \\
{[\mathrm{Ne} I I I]}\end{array}\right\}$ & 10.3 & 1.03 & 14 & 52 \\
\hline 3889.1 & $\mathrm{H} \zeta\}$ & & & & \\
\hline 3888.6 & HeI & 4.3 & 0.47 & 6 & 20 \\
\hline 3869.7 & [NeIII] & 19.3 & 2.12 & 29 & 120 \\
\hline $\begin{array}{l}3728.9 \\
3726.2\end{array}$ & $\begin{array}{l}{[\mathrm{O} \mathrm{u}]} \\
{[\mathrm{Ou}]}\end{array}$ & 28.7 & 3.75 & 51 & 35 \\
\hline $\begin{array}{l}3726.2 \\
3425.8\end{array}$ & $\begin{array}{l}[\mathrm{OIII}]) \\
{[\mathrm{NeV}]}\end{array}$ & 14.5 & 2.46 & 33 & 130 \\
\hline
\end{tabular}


smooth. In the case of NGC 3516, in which Seyfert found very great broadening, with a half-width of some $4000 \mathrm{~km} \mathrm{~s}^{-1}$, Souffrin (1969) suggested that the very broad $H$ wings might be produced by Doppler broadening in an inner region of higher density $\left(N_{e} \geqslant 10^{8} \mathrm{~cm}^{-3}\right)$, in which the (less broad) forbidden lines cannot appear because of the high density. [O III] $\lambda 4363$, of intermediate width, would be produced in an intermediate region with lower turbulent velocities where $N_{e} \simeq 10^{6} \mathrm{~cm}^{-3}$, and the other, narrower, forbidden lines would arise in an outer region where $N_{e} \approx 10^{4} \mathrm{~cm}^{-3}$.

Whatever the main broadening agent is found to be, extreme lack of homogeneity in the emitting region must be an important factor, and all those who have worked on the physical conditions in Seyfert nuclei have arrived at this conclusion. Weymann (1970) suggested that $N_{e}$ as high as $3 \times 10^{9} \mathrm{~cm}^{-3}$ might exist in an inner core of radius $7.5 \times 10^{15} \mathrm{~cm}$, in which only the Balmer wings would be produced, and here electron scattering might indeed be important.

\subsubsection{Absorption Lines in Gas in NGC 4151}

One of the very interesting discoveries, and one that links the Seyfert nuclei with QSOs, has been the multiple-redshift absorption lines of $\mathrm{H}$ and $\mathrm{He}$ I found by Anderson and Kraft (1969) in coudé spectra of the nucleus of NGC 4151, and their variability discovered by Cromwell and Weymann (1970). Anderson and Kraft found that $\mathrm{He}$ I $\lambda 3889$ appeared with three narrow components, giving velocities (relative to the nucleus itself) of $-280,-550$, and $-840 \mathrm{~km} \mathrm{~s}^{-1}$; weaker components were also visible in the Balmer lines, the largest velocity differences being $-970 \mathrm{~km} \mathrm{~s}^{-1}$ at $\mathrm{H} \beta$. These lines can best be interpreted as arising in gas ejected from the nucleus of NGC 4151, which is known to be variable in optical light. Further, Cromwell and Weymann found that these components are variable and can appear and disappear with a time scale of order one year. Osterbrock (1970) estimated that the gas outflow suggested by these observations amounts to about $\frac{1}{2} M_{\odot}$ per year.

\subsubsection{Emission-Line Variability}

Variability on a longer time scale was found by Andrillat and Souffrin (1967) in the emission-line spectrum of NGC 3516. Their spectra, obtained in 1967, showed that a definite change had occurred since the observations by Seyfert in 1943. The Balmer lines had almost disappeared, and the forbidden lines had strengthened. They suggested that matter ejected by the active inner nucleus had expanded and become less dense. It will be very interesting to carry out long-term monitoring of the spectra of a variety of Seyfert nuclei, and see whether others vary in this way, because an idea of the size of the variable regions can be provided by detailed study of such variations.

\subsection{N-GALAXIES}

The original isolation and classification of $\mathrm{N}$-type galaxies was the form classification in Morgan's classical paper of 1958. He defined these galaxies as "systems having small brilliant nuclei superposed on a considerably fainter background". In that paper 
he classified NGC 4051 and 4151, two of the original Seyfert galaxies, as N systems. Later, in the paper on radio galaxies by Matthews et al. (1964), N-type galaxies were defined as "galaxies having brilliant star-like nuclei containing most of the luminosity of the system. A faint nebulous envelope of small visible extent is observed". It was realized in that paper that many radio galaxies fall into this category, and it was also pointed out that these objects may be related to the compact galaxies discovered by Zwicky.

In practice, the term ' $\mathrm{N}$-galaxy' has been most often used for radio galaxies conforming to the description by Matthews et al. Now that a specific classification scheme has been described by Morgan (this volume, p. 97) with 3 basic classes and 2 subclasses, it is to be hoped that there will be more precision in the allotment of new objects into appropriate classes, and that this will replace the arbitrary naming of some objects as 'Seyfert galaxies' and some as 'N-galaxies', which has led to some confusion.

As spectroscopic information has accumulated for objects that have been given a form classification as $\mathrm{N}$-type systems, it has become apparent that a wide variety of types of line spectra are possible. These range from objects with strong and very broad emission lines, just like those described in Section 2.1, objects with narrower but still intensely strong emission lines, and objects with much weaker emission lines, and even with absorption lines only. Lynds (1968a) has discussed this variety of spectra. It is probable that a more careful form classification of objects with absorption lines only would place them in Morgan's class $\mathrm{C}$, and then my physical division would allot them to type (3) of the compacts, to be described in Section 4.

Two good examples of $\mathrm{N}$-type radio galaxies are $3 \mathrm{C} 234$ and $3 \mathrm{C} 445$, and the emission lines and their estimated relative strengths were given by Schmidt (1965a) in his paper on redshifts of 31 radio galaxies. Table III, taken from G. Burbidge (1970), lists the spectrum lines seen in $18 \mathrm{~N}$ galaxies identified with radio sources.

An interesting question concerns the relative contribution to the emitted light coming from stars, from hot gas, and from non-thermal radiation. Since my discussion concerns only the line spectra, the important question is whether or not absorption lines coming from the stellar component are visible in the spectrum. Since the non-thermal component of the continuum emission may be variable, as in the N-galaxy 3C 371, absorption lines may be visible at times and disappear when the starlight is much exceeded by the thermal radiation from hot gas and synchrotron radiation.

Two N-type galaxies have been found to display a phenomenon which may relate to the double redshift of NGC 1275 . We describe these objects next.

\section{$3 C 227$ and $3 C 390.3$}

These two objects are listed as N-galaxies by Wyndham (1965); 3C 227 was described by Matthews et al. (1964) and Zwicky had informed Wyndham that 3C 390.3 was very compact, only just discernible as extended with the 200-in. telescope, having a redshift velocity of about $17000 \mathrm{~km} \mathrm{~s}^{-1}$. Lynds (1968b) described their spectra, 
TABLE III

Spectroscopic properties of $\mathbf{N}$ galaxies identified with radio sources

\begin{tabular}{|c|c|c|}
\hline Object & $z$ & Lines \\
\hline \multicolumn{3}{|l|}{ MSH $05-43$} \\
\hline (Pictor A) & 0.0342 & {$\left[\mathrm{OII}_{11}\right] 3727,[\mathrm{NeII}] \lambda 3869, \mathrm{H} \delta, \mathrm{H} \gamma, \mathrm{H} \beta,[\mathrm{OII}] \lambda \lambda 4363,4959,5007$.} \\
\hline 3C 371 & 0.0508 & [OII] $] 23727,[\mathrm{OIII}] \lambda \lambda 4959,5007$, CaII $\mathrm{H}$ and $\mathrm{K}$ abs. \\
\hline $3 \mathrm{C} 445$ & 0.0568 & $\begin{array}{l}{[\mathrm{Nev}] \lambda 3426,[\mathrm{OII}] \lambda 3727,[\mathrm{NeIII}] \lambda \lambda 3869,3968,\left[\mathrm{~S}_{\mathrm{II}}\right] \lambda \lambda 4068,4076,} \\
\mathrm{H} \delta, \mathrm{H} \gamma, \mathbf{H} \beta,[\mathrm{OII}] \lambda \lambda 4363,4959,5007 \text {. No abs. }\end{array}$ \\
\hline $3 \mathrm{C} 390.3^{\mathrm{a}}$ & 0.0569 & $\begin{array}{l}{[\mathrm{Nev}] \lambda 3426,[\mathrm{OII}] \lambda 3727,[\mathrm{NeIII}] \lambda \lambda 3869,3968, \mathrm{H} \delta, \mathrm{H} \gamma, \mathrm{H} \beta,} \\
{[\mathrm{OIII}] \lambda \lambda 4363,4959,5007, \mathrm{H} \alpha . \text { No abs. }}\end{array}$ \\
\hline PKS $0521-36$ & 0.061 & $\begin{array}{l}{[\mathrm{Nev}] \lambda \lambda 3346,3426,[\mathrm{OII}] \lambda 3727,[\mathrm{Ne} \mathrm{II}] \lambda \lambda 3869,3968, \mathrm{H} \delta, \mathrm{H} \gamma,} \\
{[\mathrm{OIII}] \lambda \lambda 4959,5007 .}\end{array}$ \\
\hline $3 \mathrm{C} 227^{\mathrm{b}}$ & 0.0855 & $\begin{array}{l}{[\mathrm{Nev}] \lambda 3426,[\mathrm{OII}] \lambda 3727,[\mathrm{NeIII}] \lambda \lambda 3869,3968, \mathrm{H} \gamma, \mathrm{H} \beta} \\
{[\mathrm{OIII}] \lambda \lambda 4363,4959,5007, \mathrm{HeI} \lambda 5876, \mathrm{H} \alpha . \text { No abs. }}\end{array}$ \\
\hline PKS $1417-19$ & 0.1192 & $\begin{array}{l}{[\mathrm{O} I 1] \lambda 3727,[\mathrm{NeIII}] \lambda 3869,[\mathrm{OIII}] \lambda \lambda 4363,5007, \mathrm{H} \gamma, \mathrm{H} \beta, \mathrm{H} \alpha \text {. }} \\
\text { No abs. }\end{array}$ \\
\hline PKS 2300- 18 & 0.129 & {$[\mathrm{NeIII}] \lambda 3869, \mathrm{H} \gamma, \mathrm{H} \beta,[\mathrm{O} I 1 \mathrm{I}] \lambda \lambda 4363,4959,5007$.} \\
\hline PKS $1340+05$ & 0.1333 & {$[\mathrm{OII}] \lambda 3727, \mathrm{CaII} \mathrm{H}$ and $\mathrm{K}$ abs. } \\
\hline PKS 2349-01 & 0.174 & {$[\mathrm{OII}] \lambda 3727, \mathbf{H} \delta, \mathbf{H} \gamma, \mathbf{H} \beta,[\mathrm{OIII}] \lambda \lambda 4363,4959,5007$.} \\
\hline $3 \mathrm{C} 234$ & 0.1846 & $\begin{array}{l}{[\mathrm{Nev}] \lambda \lambda 3346,3426,\left[\mathrm{O}_{\mathrm{II}}\right] \lambda 3727,[\mathrm{NeIII}] \lambda \lambda 3869,3968, \mathrm{H} \zeta, \mathrm{H} \delta, \mathrm{H} \gamma,} \\
{\left[\mathrm{O}_{\mathrm{III}}\right] \lambda \lambda 4363,4959,5007, \mathrm{He} \text { II } \lambda \lambda 3203,4686, \mathrm{H} \beta . \text { No abs. }}\end{array}$ \\
\hline $3 C 287.1$ & 0.2156 & $\begin{array}{l}{[\mathrm{Nev}] \lambda 3426,[\mathrm{OII}] \lambda 3727,[\mathrm{NeIII}] \lambda \lambda 3869,3968, \mathrm{H} \beta,[\mathrm{OIII}] \lambda \lambda 4959 \text {, }} \\
5007 .\end{array}$ \\
\hline $3 \mathrm{C} 17$ & 0.2201 & {$[\mathrm{OII}] \lambda 3727,[\mathrm{NeII}] \lambda 3869, \mathrm{H} \beta,[\mathrm{OII}] \lambda \lambda 4959,5007$.} \\
\hline $3 C 459$ & 0.2205 & $\begin{array}{l}{[\mathrm{Nev}] \lambda 3426,[\mathrm{OII}] \lambda 3727,[\mathrm{NeIII}] \lambda 3869,[\mathrm{O} \text { III }] \lambda \lambda 4959,5007 .} \\
\text { Weak em.; no abs.? }\end{array}$ \\
\hline 3C 171 & 0.2387 & {$[\mathrm{OII}] \lambda 3727,[\mathrm{NeIII}] \lambda \lambda 3869,3968,[\mathrm{OIII}] \lambda \lambda 4363,4959,5007, \mathrm{H} \beta$.} \\
\hline $3 C 79$ & 0.2561 & $\begin{array}{l}{[\mathrm{Nev}] \lambda \lambda 3346,3426,[\mathrm{OII}] \lambda 3727,[\mathrm{NeIII}] \lambda \lambda 3869,3968, \mathrm{H} \gamma,} \\
{[\mathrm{OII}] \lambda \lambda 4363,4959,5007, \mathrm{He} \text { II } 4686, \mathrm{H} \beta .}\end{array}$ \\
\hline $3 C 109$ & 0.3056 & $\begin{array}{l}{[\mathrm{Nev}] \lambda 3426,[\mathrm{OII}] \lambda 3727,[\mathrm{NeIII}] \lambda \lambda 3869,3968, \mathrm{H} \gamma, \mathrm{H} \beta} \\
{[\mathrm{OIII}] \lambda \lambda 4363,4959,5007 .}\end{array}$ \\
\hline 3C 177 & no publ. $z$ & No emission lines, Ca II $\mathrm{H}, \mathrm{K}$, and $\mathrm{G}$ band abs. \\
\hline
\end{tabular}

a Balmer emission lines very broad, with two maxima.

b Balmer emission lines double.

and pointed out that $3 \mathrm{C} 227$ has hydrogen emission lines that are clearly double, and the lines in 3C 390.3 are fully as wide as the total redshift. The forbidden lines in $3 \mathrm{C} 227$, among which [Nev] $\lambda 3426$ is very prominent, are single and agree in redshift with the longer-wavelength component of the hydrogen lines.

In 3C 390.3, we have found that the extremely broad emission lines of $\mathrm{H}$ are also double, although the two maxima are not as distinct as in 3C 227. Again, the forbidden lines are single and give the same redshift as the longer-wavelength component of hydrogen. Measurements on our Lick spectrograms give:

Mean recession velocity, longward components of $\mathrm{H} \alpha, \mathrm{H} \beta+$ forbidden lines (this component of $\mathrm{H} \gamma$ is blended with [O III] 24363$) \ldots=16800 \mathrm{~km} \mathrm{~s}^{-1}$; Mean recession velocity, shortward components of $\mathrm{H} \alpha, \mathrm{H} \beta, \mathrm{H} \gamma \ldots=12250 \mathrm{~km} \mathrm{~s}^{-1}$. Thus the velocity difference is $4550 \mathrm{~km} \mathrm{~s}^{-1}$, exceeding the difference of $3000 \mathrm{~km} \mathrm{~s}^{-1}$ seen in NGC 1275. Possibly this phenomenon may represent an early stage in the develop- 
ment of structures that later may resemble what is seen in NGC 1275. The absence of forbidden lines in the shortward component suggests that the electron density is at present higher in the gas giving rise to this emission.

\subsection{COMPaCtS OF Class (1)}

We turn now to my physical grouping, class (1) of the Zwicky compact objects. After the objects are picked on the basis of their compact nature, this grouping is made solely according to the spectrographic information, and objects falling into this category resemble classical Seyfert galaxies in having very broad strong hydrogen lines in their spectra. However, they have larger redshifts than the classical Seyfert galaxies. Known examples are listed in Table I.

Three good examples of this class are III $\mathrm{Zw} 0008+10$, for which Arp (1968) published a direct photograph and the spectrum $(z=0.089)$, I $\mathrm{ZW}_{\mathrm{W}} 051+12$, with $z=0.061$, studied by Sargent (1968a), and II Zw $2130+09$, with $z=0.061$, also studied by Sargent (1968b). The spectrum of III $\mathrm{Zw} 0008+10$ has very broad hydrogen lines, and narrow forbidden lines of [OII], [OIII], and [NeIII]. In view of the clearly-defined doubling of the hydrogen lines in 3C 227 and 3C 390.3, it is interesting that Arp noted that doubling in $\mathrm{H} \beta$ and $\mathrm{H} \delta$ was discernible in the spectrum of III $\mathrm{Zw} 0008+10$, and $\mathrm{H} \gamma$ could be seen on microphotometer tracings to be double.

I $\mathrm{Zw} 0051+12$ and II Zw $2130+09$ are interesting because they both have strong broad permitted lines of FeII in their spectra. The former has no forbidden lines at all; the electron density must be greater than $10^{6} \mathrm{~cm}^{-3}$ and Sargent's spectrophotometric measurements then yielded $R \leqslant 5 \times 10^{17} \mathrm{~cm}$ for the dimension of the lineemitting region. The object II $\mathrm{Zw} 2130+09$ does have forbidden lines, but they are weaker than usual so the electron density is probably fairly high. The FeII emission lines in these two objects provide an interesting link between QSOs and compact galaxies of this class; these same FeII lines were seen in the spectrum of the QSO $3 \mathrm{C}$ 273, which is known to have a high electron density because the forbidden lines of [OIII] are not seen in its spectrum.

Finally, we may note that conversion of the redshifts of these compact galaxies to distances, using a Hubble constant of $75 \mathrm{~km} \mathrm{~s}^{-1} \mathrm{Mpc}^{-1}$, yields photographic absolute magnitudes of $-22.6,-23.2$, and -22.8 , respectively. This is a considerably higher luminosity than that of normal bright elliptical galaxies.

\subsection{REDSHIFTS AND THEIR DISTRIBUTION IN THE SKY FOR COMPACT OBJECTS}

We may ask whether there is anything unusual in the distribution of redshifts of those compact objects characterized by non-thermal emission, or whether there is anything in their distribution in the sky in relation to other galaxies, to indicate that their redshifts might not be due to expansion of the Universe.

The existence of a peak at $z=0.061$ in a histogram plotting numbers of objects at steps of 0.01 in redshifts was pointed out by Burbidge (1968) and Burbidge and Burbidge (1969). This might mean fluctuations in the recent past in the occurrence of such objects, or a non-cosmological origin for the redshifts, but no explanation 
or interpretation is available. We await the measurement of more redshifts to see whether the peak is maintained, whether it grows, or whether it disappears.

The occurrence of compact objects near galaxies can also be looked for. The radio source OQ 208 was thought to be identified with a star-like object $10^{\prime \prime}$ from a brighter galaxy (Ryle and Pooley, 1969), but spectroscopic observations at various observatories, including my own observations at Lick, revealed that the star-like object is in fact a normal star, and the spectrum of the galaxy is a good example of the Seyfert or compact class (1) type, with broad strong emission lines and a redshift $z=0.077$.

Weedman (1970) found that Markarian 205 is a compact galaxy with a spectrum like that of typical Seyfert galaxies and a redshift $z=0.070$, and it lies only 40 " from the center of the normal spiral galaxy NGC 4319, well within the spiral arms of that galaxy. Lick spectrograms of NGC 4319 show that it has a recession velocity of some $+1500 \mathrm{~km} \mathrm{~s}^{-1}$, i.e. $z=0.005$.

We are reminded of the fact that two QSOs also lie very near spiral galaxies: 3C 275.1 is near NGC 4651 (Sandage et al., 1965), and the radio-quiet object QS $1108+285(z=2.192)$ lies about 1 arc min from NGC 3561 (Stockton 1969), the galaxy long known to have a compact appendage in the form of the 'Ambartsumiam knot'.

\section{Compacts of Class (2)}

\subsection{General discussion}

The spectroscopic observations of Zwicky compacts by Sargent (1970b) show that objects with sharp emission lines are most numerous. Also the Markarian and Haro galaxies, with strong UV continua, are mostly of this type (see references given in Section 1). The range of ionization and excitation exhibited by the emission lines is like that of bright $\mathrm{HII}$ regions in our own and other galaxies, and the presumption is strong that the source of energy is a large number of massive young $O B$ stars.

Objects of this type have a great range in size, luminosity, and morphological appearance. Direct photographs of several, and a typical spectrum, are reported by Sargent (1970b). Some objects have jets; some are in the form of rings with no central nucleus; most are irregular.

An interesting example of an extremely small compact double galaxy with sharp emission lines in its spectrum was described by Arp (1965). The recession velocity is only $+1326 \mathrm{~km} \mathrm{~s}^{-1}$, yet the apparent photographic magnitudes of the two components are 17.8 and 17.9 , and their separation and respective diameters are only about 1 arc sec. Thus the absolute magnitudes are only -12.7 and -12.8 (for $H=100 \mathrm{~km} \mathrm{~s}^{-1} \mathrm{Mpc}^{-1}$ ) and the diameter of each component is only $70 \mathrm{pc}$. The nature and evolutionary history of such an object is very puzzling, and since it was discovered by chance on a plate taken with the Palomar 200-in. telescope, the space density of galaxies of this sort might be quite high. A similar faint galaxy was discovered by Kinman (1965) in an investigation of the fainter Haro-Luyten blue stellar 
objects; it has sharp emission lines, a small redshift, and an absolute $\mathbf{B}$ magnitude of about -14 and was just detectable as non-stellar on Kinman's photograph taken with the Lick 120-in. telescope.

\subsection{Possible young Galactic NUClei}

Sargent (1970c) drew attention to a particular kind of dwarf compact galaxy with sharp emission lines and $21-\mathrm{cm}$ radiation, which he and Searle are studying and which they think might be young recently-formed objects. The three examples of this type for which they have observations are II Zw $0553+03$, I Zw $0930+55$, and I $\mathrm{Zw} 1531+46$. These have small redshifts and values of $M_{p}$ fainter than -17 . The 21-cm observations are by Chamaraux et al. (1970); see also Heidmann (this volume, p. 264). The structures suggest that these objects may consist of giant $\mathrm{O}$-associations embedded in neutral hydrogen, although at the moment the $21-\mathrm{cm}$ detection is certain in only one of the three objects. Sargent and Searle conclude that they are either young or have only recently formed massive stars (the observations do not exclude the possibility that a substructure of faint red stars may be present). Because of the way in which these galaxies were discovered, it is clear that they may be very frequent in space.

\section{Compacts of Class (3)}

In this class of compact galaxy the spectra display only absorption lines, typically CaII, the G-band, and the $\mathrm{Mg} b$ feature, though a few show Balmer lines as well as CaII. The colors are like those of elliptical galaxies, and it is clear that the objects are dense groupings of stars of the population type normally taken to be some $10^{10} \mathrm{yr}$ old. Their evolutionary history is therefore of great interest: what has caused them to have a different structure from that of normal elliptical galaxies?

The best-known example of this kind of galaxy is NGC 4486-B, the companion of M87. Rood (1965) measured its brightness distribution and, using the large velocity dispersion measured by Minkowski (1961), found that it has a mass of $5 \times 10^{10} M_{\odot}$ and a high mass-to-light ratio of 80 . He suggested that its small compact size might be due to tidal limitation by the extensive and very massive M87.

There are other compact galaxies of this kind, however, for which the suggestion of tidal limitation cannot be made. Zwicky (1968) discovered two clusters of compact galaxies, $\mathrm{Zw} \mathrm{C1} 0152+33$ and $\mathrm{Zw} \mathrm{C1} \mathrm{1710+64,} \mathrm{and} \mathrm{the} \mathrm{question} \mathrm{of} \mathrm{their} \mathrm{relation} \mathrm{to}$ clusters of normal galaxies is not at present understood. Sargent (1971) has studied the virial relation between kinetic and potential energy for $\mathrm{Zw} \mathrm{C1} 0152+33$.

\section{Quasi-Stellar Objects}

I shall cover only the emission-line spectra of QSOs; Lynds will discuss the very interesting absorption-line spectra, and I have time only for a brief and condensed account. A fuller description of both emission and absorption line spectra was given earlier this year (E. M. Burbidge, 1970). 


\subsection{IDENTIFICATION OF EMISSION LINES}

The emission lines found in spectra of QSOs are those expected to be produced in a hot gas at fairly low density, with a chemical composition similar to that of the Sun and the stars and gaseous nebulae in the solar neighborhood. This was shown by the first line identifications made by Schmidt (1965b), in which emission lines predicted to be strong in the far ultraviolet spectral region in planetary nebulae (Osterbrock, 1963) were found to be present, with very large redshifts, in the spectra of QSOs.

Table IV, taken from E. M. Burbidge (1970), lists most of the emission lines that have been found with rest wavelengths in the range 1200-5000 $\AA$ (weaker features seen rarely are not included). A large range of ionization is found, from $7.6 \mathrm{eV}$

TABLE IV

Principal emission lines seen in spectra of QSOs, $1200-5000 \AA$

\begin{tabular}{|c|c|c|c|}
\hline Ident. & $\lambda(\mathrm{A})$ & Ident. & $\lambda(\mathrm{A})$ \\
\hline $\mathbf{L} \gamma-\alpha$ & $1216^{a}$ & MgII & $2798^{a}, \mathrm{~b}$ \\
\hline Nv & $1240^{b}$ & [Ariv] & 2854,2869 \\
\hline Sirv & $1397^{\mathrm{b}}$ & {$[\mathrm{Mgv}]$} & 2931 \\
\hline OIv] & 1406 & HeII & 3203 \\
\hline Civ & $1549^{a, b}$ & {$[\mathrm{Nev}]$} & 3346,3426 \\
\hline HeII & 1640 & [OII] & $3727^{b}$ \\
\hline OIII] & 1664 & [NeIII] & 3869,3968 \\
\hline CIII] & $1909^{a}$ & [OIII] & $4363,4959,5007^{a}$ \\
\hline $\mathrm{CII}]$ & 2326 & Balmer series & $4102,4340,4861^{a}$ \\
\hline
\end{tabular}

a Strongest transitions.

b Blends of doublets.

which is required for single ionization of $\mathrm{Mg}$, to $97 \mathrm{eV}$ required to produce $\mathrm{Ne}^{+4}$ or $109 \mathrm{eV}$ for $\mathrm{Mg}^{+4}$ (or even $186 \mathrm{eV}$ for $\mathrm{Mg}^{+6}$ since [Mg VII] has been identified in spectra of a few QSOs. A useful table which groups the lines according to electron configuration in the emitting ions was published by Lynds (1968b). This lists all possible transitions between about 1000 and $7000 \AA$, in the various ionization stages of all elements having a solar-neighborhood ('normal') relative abundance greater than $10^{-6}$ hydrogen, and those transitions which have actually been observed are noted. Burbidge and Burbidge (1967) gave a compilation of emission lines observed in 60 QSOs, with eye estimates of intensity, and the results for recent measurements of line intensities in 28 QSOs were given by Oke et al. (1970).

\subsection{Physical conditions in the Line-emitting Region}

Following the first study of the physics of the line-emitting region in $3 \mathrm{C} 273$ and 3C 48 (Greenstein and Schmidt, 1964), and the study of average conditions in a composite of several QSOs (Osterbrock and Parker, 1966), it has become increasingly apparent that, as in the Seyfert galaxies, the line-emitting region must be non-homogeneous and probably consists of a combination of stratified layers and denser clouds 
or filaments in a hotter, lower-density background. In these earlier studies a level of ionization was assumed. Bahcall and Kozlovsky (1969), however, calculated the ionization equilibrium in $3 \mathrm{C} 273$ and $3 \mathrm{C} \mathrm{48}$, assuming that the energy for ionization comes from a continuum with a power-law distribution of the flux, $F(v)$, as a function of frequency, $v$.

Osterbrock (1969) published an updated list of calculated line strengths for the elements $\mathrm{H}, \mathrm{He}, \mathrm{C}, \mathrm{N}, \mathrm{O}, \mathrm{Ne}$, and $\mathrm{Mg}$ in 'normal' abundances, and also a further list of weaker lines from $\mathrm{Mg}, \mathrm{Si}, \mathrm{S}, \mathrm{Ar}$, some of which have been observed. A result which was apparent in the analysis of several QSOs by Osterbrock and Parker, and also in the study of 3C 273 by Bahcall and Kozlovsky where the level of ionization was calculated and not assumed, was that He appears to be underabundant in these objects, whereas the other elements, relative to hydrogen, appear normal. Further work on this is desirable.

The widths of the emission lines are generally not as great as in the spectra of Seyfert nuclei; typically they are about $50 \AA$, although the resonance lines Ly- $\alpha$ and Civ $\lambda 1549$ may be much wider. Often the forbidden lines are narrower than the permitted lines, as in some Seyfert nuclei, and this led Burbidge et al. (1966) to propose a model in which electron scattering is the broadening agent, in a stratified medium where the various lines are produced in different regions. It is not known whether this mechanism, or Doppler broadening by random or organized motion, is responsible for the line broadening.

The discovery by Lynds and Wills (1970) of 4C 5.34, a QSO with the very large redshift of $z=2.877$, has made possible the study of the $\mathrm{Ly}-\alpha / \mathrm{Ly}-\beta$ intensity ratio, a quantity pointed out by Bahcall (1966) to be of interest, and the Lyman continuum. Ly- $\beta$ can also be studied in 5C 2.56, which has $z=2.388$; this latter QSO, which had become very faint, recently brightened so that I was able again to obtain spectrograms at Lick and to confirm a tentative earlier measurement of its redshift.

Finally, I would like to draw attention to the fact that, despite their similarities, there are distinct spectroscopic differences between the QSOs, as was seen in the first quantitative study of $3 \mathrm{C} 273$ and 3C 48. For example, the emission line NV $\lambda 1240$ may not appear at all in some QSOs of large redshift, while in others it may be almost as strong as Ly- $\alpha$ emission, as is the case in PHL 3424 (Sandage and Luyten, 1967) and in 5C 2.56. Quantitative study of these and other differences can now be undertaken.

\section{Acknowledgement}

Extragalactic research at UCSD is supported by the National Science Foundation and by NASA under grant NGL-05-005-004.

\section{References}

Ambartsumian, V. A.: 1958, in Solvay Conference on Structure and Evolution of the Universe, (ed. by

R. Stoops, Brussels), p. 241. 
Ambartsumian, V. A.: 1965, in Solvay Conference on Structure and Evolution of Galaxies, Interscience, John Wiley and Sons Ltd., London, p. 1.

Ambartsumian, V. A.: 1970, Nuclei of Galaxies, Study Week, Pontifical Academy of Sciences, in press.

Anderson, K. S. and Kraft, R. P.: 1969, Astrophys. J. 158, 859.

Andrillat, Y. and Souffrin, S.: 1967, Astrophys. Letters 1, 111.

Arakelian, M. A., Dibai, E. A., and Yesipov, V. F.: 1970, Astrofizika 6, 39.

Arp, H. C.: 1965, Astrophys. J. 142, 402.

Arp, H. C.: 1966, Atlas of Peculiar Galaxies, Cal. Tech. Book Store, Pasadena.

Arp, H. C.: 1968, Astrophys. J., 152, 1101.

Arp, H. C., Khachikian, E. E., Lynds, C. R., and Weedman, D.: 1968, Astrophys. J. Letters 152, L103.

Bahcall, J.: 1966, Astrophys. J. 145, 684.

Bahcall, J. and Kozlovsky, B. Z.: 1969, Astrophys. J. 155, 1077; Astrophys. J. 158, 529.

Burbidge, E. M.: 1970, Nuclei of Galaxies, Study Week, Pontifical Academy of Sciences, in press.

Burbidge, G. R.: 1968, Astrophys. J. Letters 154, L41.

Burbidge, G. R.: 1970, Ann. Rev. Astron. Astrophys. 8, 369.

Burbidge, G. R. and Burbidge, E. M.: 1965, Astrophys. J. 142, 1351.

Burbidge, G. R. and Burbidge, E. M.: 1967, Quasi Stellar Objects, W. H. Freeman, San Francisco.

Burbidge, G. R. and Burbidge, E. M.: 1969, Nature 222, 735.

Burbidge, G. R., Burbidge, E. M., Hoyle, F., and Lynds, C. R.: 1966, Nature 210, 774.

Burbidge, G. R., Burbidge, E. M., and Prendergast, K. H.: 1959, Astrophys. J. 130, 26.

Burbidge, G. R., Burbidge, E. M., and Prendergast, K. H.: 1963, Astrophys. J. 137, 1022.

Burbidge, G. R., Burbidge, E. M., and Sandage, A. R.: 1963, Rev. Mod. Phys. $35,947$.

Chamaraux, P., Heidmann, J., and Lauqué, R.: 1970, Astron. Astrophys., in press.

Cromwell, R. and Weymann, R. J.: 1970, Astrophys. J. Letters 159, L147.

Danielson, R. E., Savage, B. D., and Schwarzschild, M.: 1968, Astrophys. J. Letters 154, L117.

Dibai, E. A. and Pronik, V. I.: 1965, Astrofizika 1, 78.

Dibai, E. A. and Pronik, V. I.: 1966, Izv. Krymsk. Astrofiz. Obs. 35, 87.

DuPuy, D. L.: 1968, Publ. Astron. Soc. Pacific 80, 29.

Greenstein, J. L. and Schmidt, M.: 1964, Astrophys. J. 140, 1.

Haro, G.: 1956, Bol. Obs. Tonantzintla Tacubaya 2, No. 14, 8.

Kinman, T. D.: 1965, Astrophys. J. 142, 1241.

Lynds, C. R.: 1968a, Astron. J. 73, 888.

Lynds, C. R.: 1968b, in S. Bashkin (ed.), Proc. Conf. on Beam-Foil Spectroscopy; Gordon and Breach, New York, p. 539.

Lynds, C. R.: 1970, Astrophys. J. Letters, 159, L151.

Lynds, C. R. and Wills, D.: 1970, Nature 226, 532.

Markarian, B. E.: 1967, Astrofizika 3, 55.

Markarian, B. E.: 1969, Astrofizika 5, 443, 581.

Matthews, T. A., Morgan, W. W., and Schmidt, M.: 1964, Astrophys. J. 140, 35.

Minkowski, R.: 1957, in H. C. van de Hulst (ed.), 'Radio Astronomy', IAU Symp. 4, 107.

Minkowski, R.: 1961, in G. C. McVittie (ed.), 'Problems of Extragalactic Research', IAU Symp. $15,112$.

Minkowski, R.: 1968, Astron. J. 73, 842.

Morgan, W. W.: 1958, Publ. Astron. Soc. Pacific 70, 364.

Morgan, W. W.: 1970, Nuclei of Galaxies, Study Week, Pontifical Academy of Sciences, in press.

Oke, J. B., Neugebauer, G., and Becklin, E.: 1970, Astrophys. J. 159, 341.

Oke, J. B. and Sargent, W. L. W.: 1968, Astrophys. J. 151, 807.

Osterbrock, D. E.: 1963, Planetary Space Sci. 11, 621.

Osterbrock, D. E.: 1969, Mem. Soc. Roy. Sci., Liège. Ser. V. 16, 391.

Osterbrock, D. E.: 1970, Nuclei of Galaxies, Study Week, Pontifical Academy of Sciences, in press.

Osterbrock, D. E. and Parker, R. A. R.: 1965, Astrophys. J. 141, 892.

Osterbrock, D. E. and Parker, R. A. R.: 1966, Astrophys. J. 143, 268.

Pacholczyk, A. G. and Weymann, R.: 1968, Astron. J. 73, 836. 
Rood, H. J.: 1965, Astron. J. 70, 689.

Rubin, V. C. and Ford, W. K.: 1968, Astrophys. J. 154, 431.

Ryle, M. and Pooley, G. G.: 1969, Astrophys. Letters 4, 137.

Sandage, A. R. and Luyten, W. J.: 1967, Astrophys. J. 148, 767.

Sandage, A. R., Véron, P., and Wyndham, J.: 1965, Astrophys. J. 142, 1307.

Sargent, W. L. W.: 1968a, Astrophys. J. Letters 152, L31.

Sargent, W. L. W.: 1968b, Astron. J. 73, 893.

Sargent, W. L. W.: 1970a, Astrophys. J. 159, 765.

Sargent, W. L. W.: 1970b, Astrophys. J. 160, 405.

Sargent, W. L. W.: 1970c, Nuclei of Galaxies, Study Week, Pontifical Academy of Sciences, in press.

Sargent, W. L. W.: 1971, Astrophys. J., in press.

Schmidt, M.: 1965a, Astrophys. J. 141, 1.

Schmidt, M.: 1965b, Astrophys. J. 141, 1295.

Seyfert, C. K.: 1943, Astrophys. J. 97, 28.

Shklovsky, I. S.: 1966, Soviet Astron. 9, 683.

Souffrin, S.: 1969, Astron. Astrophys. 1, 305, 414.

Stockton, A. N.: 1968, Astron. J. 73, 887.

Stockton, A. N.: 1968, Astrophys. J. Letters 155, L141.

de Vaucouleurs, G. and de Vaucouleurs, A.: 1968, Astron. J. 73, 858.

Vorontsov-Velyaminov, B. A.: 1959, Atlas and Catalogue of Interacting Galaxies, Moscow.

Walker, M. F.: 1966, in M. Arakeljan (ed.), 'Non-Stable Phenomena in Galaxies', IAU Symp. 29, 21.

Walker, M. F.: 1968, Astrophys. J. 151, 71.

Weedman, D. W.: 1970, Astrophys. J. Letters 161, L113.

Weedman, D. W. and Khachikian, E. K.: 1968, Astrofizika 4, 587.

Weedman, D. W. and Khachikian, E. K.: 1969, Astrofizika 5, 113.

Weymann, R. J.: 1970, Astrophys. J. 160, 31.

Williams, R. E. and Weymann, R. J.: 1968, Astron. J. 73, 456.

Wyndham, J.: 1965, Astrophys. J. 144, 459.

Zwicky, F.: 1964, Astrophys. J. 140, 1467.

Zwicky, F.: 1966, Astrophys. J. 143, 192.

Zwicky, F.: 1964-1968, privately circulated lists of 2000 compact galaxies available on request to the California Institute of Technology.

Zwicky, F.: 1968, Compt. Rend. Acad. Sci. Paris 266, 103.

Zwicky, F. and Humason, M. L.: 1961, Astrophys. J. 133, 794.

\section{Discussion}

Pastoriza: A drastic change in the strength of $\mathrm{H} \beta$ emission in NGC 1566 was detected at Córdoba in 1969. When the Seyfert characteristics were first observed in the nucleus of NGC 1566 by de Vaucouleurs at Mt. Stromlo in 1956, $\mathrm{H} \beta$ was broad $\left(\sim 3000 \mathrm{~km} \mathrm{~s}^{-1}\right)$ with a narrow core and the line was much stronger than the [OIII] lines. The situation was still very much the same in 1952 when Shobbrook confirmed this result, also at Mt. Stromlo. However, in several spectrograms taken at Córdoba in 1969, $\mathrm{H} \beta$ has almost vanished while the [O III] emission lines are still strong, indicating that the variation in $\mathrm{H} \beta$ cannot be explained by an increase in the continuum. The new observations are reported in Astrophysical Letters (in press).

Tovmasjan: Recently Arakelian, Dibai, Yesipov and Markarian have observed 120 galaxies from Markarian's second and third lists and discovered 18 galaxies with spectra characteristic of Seyfert galaxies - broad emission hydrogen lines and narrow forbidden lines. Results for some of them (including Markarian 205) have already been published.

Weymann: The [FexIV] identification in NGC 4151 should be regarded as uncertain. On plates taken by Mme. Souffrin, the feature was identified as [Cav] 5309, and the same identification was made independently by Alloin and Weymann at Steward Observatory.

NGC 1068 should not be regarded as a case of an object having 'both broad hydrogen and broad forbidden lines' since the extremely broad hydrogen wings characteristic of most of the Seyfert galaxies are lacking in this object. I would like to ask Mrs Burbidge if she knows of any cases where 
there are very broad hydrogen wings as well as equally broad wings on lines which are definitely [OIII] rather than [FeII].

Mrs Burbidge: I know of several cases where the widths of the hydrogen lines and the forbidden lines are comparable, and similar to NGC 1068 (and indeed similar to the QSOs, in which the majority show rather broad lines of both permitted and forbidden lines, but not the extremely extended wings shown by the $\mathrm{H}$ lines in e.g. NGC 3516 and 4151). In these cases of moderately broad profiles for both permitted and forbidden lines, the widths are of the sort shown by Walker's 'haystack' profiles in NGC 1068. I think one can say definitely that no cases are known where the forbidden lines show the extremely broad wings seen in the Balmer lines in NGC 4151. 